\title{
Control of Uncertain Time Delay System with Astatism and Parametric and Periodic Uncertainties using SSV and Factorization for Two-Degree-of-Freedom-Controller
}

\author{
Marek Dlapa ${ }^{a}$ \\ Faculty of Applied Informatics, Tomas Bata University in Zlin, Nad Stranemi 4511, 76005 Zlin, Czech Republic
}

\begin{abstract}
Keywords: Parametric Uncertainty, Structured Singular Value, Matlab Toolbox, Global Optimization, Direct Search Methods, Factorization.

Abstract: Application of the Robust Control Toolbox for Time Delay Systems with Parametric and Periodic Uncertainties Using SSV (Structured Singular Value) for the Matlab system to uncertain time delay system with astatism is performed. The D-K iteration and the algebraic approach implemented in the toolbox are applied to $2^{\text {nd }}$ order system with astatism and uncertain time delay and two other parameters in the numerator and denominator of the plant transfer function. Multiplicative uncertainty is used for treating uncertain time delay, the parametric uncertainty is modelled using general interconnection for the systems with parametric uncertainty in numerator and denominator.
\end{abstract}

\section{INTRODUCTION}

Parametric uncertainties has been an issue of robust control for several decades, the first tool was Mapping Theorem (Zadeh and Desoer, 1963) succeeded by Kharitonov Theorem (Kharitonov, 1978; Barmish, 1984 and Bialas, 1983), Edge Theorem (Bartlett et al., 1988; Barmish, 1989 and Sideris and de Gaston, 1986) and Generalized Kharitonov Theorem (Chapellat and Bhattacharyya, 1989) treating conservatism in applications to feedback loop with SISO (single-input single-out-put) controller. One of the latest results is tree structured decomposition (Barmish et al., 1989) yielding a general procedure allowing the analysis of complex closed-loop characteristic polynomials in a polynomial time and the results for specific multilinear structures (Barmish and Shi, 1990; Chapellat et al., 1993 and Fu et al., 1995) considering the closed-loop characteristic polynomials corresponding to the series connections of interval plants.

In this paper, toolbox treating parametric and periodic uncertainties using structured singular value (SSV or $\mu$, see Packard and Doyle, 1993) implementting both the algebraic approach with subsequent optimization using evolutionary algorithm
(Dlapa, 2011; Dlapa, 2018) and $D-K$ iteration as reference method is applied to $2^{\text {nd }}$ order system with astatism and uncertain time delay and parameters. The toolbox solves both parametric and dynamic uncertainties including uncertain time delay. The Robust Control Design Toolbox for Time Delay Systems with Parametric and Periodic Uncertainties Using SSV (http://dlapa.cz/homeeng.htm) deals with uncertain time delay and parametric uncertainties in the numerator and denominator of the plant transfer function. The controller is derived for two-degree-offreedom and single feedback loop (2DOF and 1DOF see Dlapa, 2014).

The controller is tuned using pole placement of nominal closed loop poles solving Diophantine equation in the ring of Hurwitz-stable and proper rational functions $\left(\mathbf{R}_{\mathrm{PS}}\right)$. The poles of the nominal closed loop are tuned via direct search methods Differential Migration (Dlapa, 2017) and NelderMead simplex method managing the issue of multimodality of the structured singular value in relationship with nominal closed loop poles. This algorithm tackles impossibility of usage of the weights with poles on imaginary axis and convergence to a global or even local minimum causing non-optimality of the resulting controller in the $D-K$ iteration (Stein and Doyle, 1991).

a https://orcid.org/ 0000-0003-2550-7062 


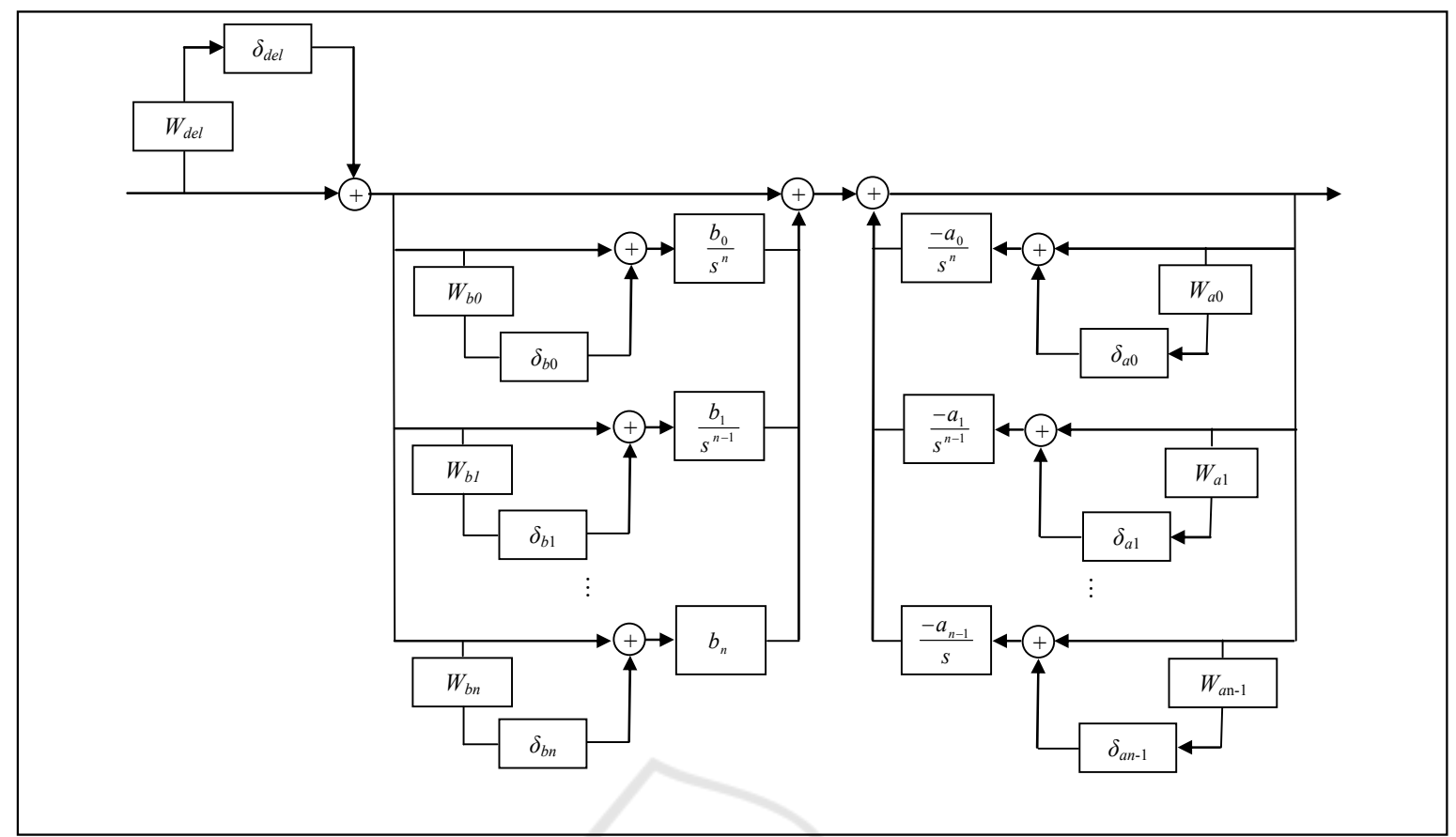

Figure 1: Modelling general parametric uncertainties system.

For reference, the controller derived using the $D$ $K$ iteration (see Doyle, 1985) is compared to the one obtained from the algebraic approach showing the pros and cons of both procedures. The resulting controllers are compared in simulations of step response for different values of time delays and periodic changes of parameters with simple feedback loop and two-degree-of-freedom structure (1DOF and 2DOF).

Notation used in the paper: $\|\cdot\|_{\infty}$ is $\mathbf{H}_{\infty}$ norm, $\bar{\sigma}(\cdot)$ denotes maximum singular value, $\mathbf{R}$ and $\mathbf{C}^{n \times m}$ are real numbers and complex matrices, respec-tively, $\overline{\mathbf{R}}_{+}$are positive real numbers, $\mathbf{I}_{n}$ is the unit matrix of dimension $n$ and $\mathbf{R}_{\mathrm{PS}}$ denotes the ring of Hurwitzstable and proper rational functions.

\section{DEFINITIONS}

Define $\Delta$ as a set of block diagonal matrices

$\Delta \equiv\left\{\operatorname{diag}\left[\delta_{1} I_{r_{1}}, \ldots, \delta_{S} I_{r_{s}}, \delta_{1} I_{c_{1}}, \ldots, \delta_{T} I_{c_{r}}, \Delta_{1}, \ldots, \Delta_{F}, \Delta_{1}, \ldots, \Delta_{K}\right]:\right.$

$\left.\delta_{s} \in \mathbf{C}, s=1 \ldots S, \delta_{t} \in \mathbf{R}, t=1 \ldots T, \Delta_{f} \in \mathbf{C}^{m_{f} \times m_{f}}, f=1 \ldots F, \Delta_{k} \in \mathbf{R}^{n_{k} \times n_{k}}, k=1 \ldots K\right\}$

where $S, T$ is the number of repeated scalar complex and real blocks,

$F, K$ is the number of full complex and real blocks,

$r_{1}, \ldots, r_{S}, \quad r_{1}, \ldots, r_{T}, \quad m_{1}, \ldots, m_{F}, \quad n_{1}, \ldots, n_{K}$ are positive integers defining dimensions of scalar and full blocks.
For consistency among all the dimensions, the following condition must be held

$$
\sum_{s=1}^{S} r_{s}+\sum_{t=1}^{T} m_{t}+\sum_{f=1}^{F} r_{f}+\sum_{k=1}^{K} m_{k}=n
$$

Definition 1: For $\mathbf{M} \in \mathbf{C}^{n \times n}$ is $\mu_{\Delta}(\mathbf{M})$ defined as

$$
\mu_{\Delta}(\mathbf{M}) \equiv \frac{1}{\min \{\bar{\sigma}(\Delta): \Delta \in \Delta, \operatorname{det}(\mathbf{I}-\mathbf{M} \Delta)=0\}}
$$

If there is no $\Delta \in \Delta$ making $\mathbf{I}-\mathbf{M} \Delta$ singular, then $\mu_{\Delta}(\mathbf{M})=0$.

\section{MODELLING OF PARAMETRIC UNCERTAINTIES FOR SSV DESIGN}

Consider general system with uncertain numerator and denominator and uncertain time delay treating parametric, periodic and time delay uncertainties:

$$
\begin{gathered}
P(s) \equiv \frac{\left(b_{0}+b_{1} s+\ldots+b_{n} s^{n}\right) e^{-\vec{s}}}{s^{n}+a_{0}+a_{1} s+\ldots+a_{n-1} s^{n-1}} \\
a_{i} \in\left[\bar{a}_{i}-A_{i}, \bar{a}_{i}+A_{i}\right], b_{i} \in\left[\bar{b}_{i}-B_{i}, \bar{b}_{i}+B_{i}\right], \\
i=0,1, \ldots, n-1, \tau \in\left[0, T_{d}\right]
\end{gathered}
$$


Time delay and parametric uncertainties vary in the predefined intervals.

$$
\left|\delta_{a i}\right|<1\left|\delta_{b i}\right|<1\left|\delta_{d e l}\right|<1
$$

And for weights $W_{a i}, W_{a i}$ and $W_{d e l}$ the following inequalities must be held for all $\omega \in \mathbf{R}$ :

$$
\begin{gathered}
W_{a i}=A_{i}, i=0,1, \ldots, n-1 \\
W_{b i}=B_{i}, i=0,1, \ldots, n \\
\left|W_{d e l}(j \omega)\right|>\left|1-e^{j \omega T_{d}}\right|
\end{gathered}
$$

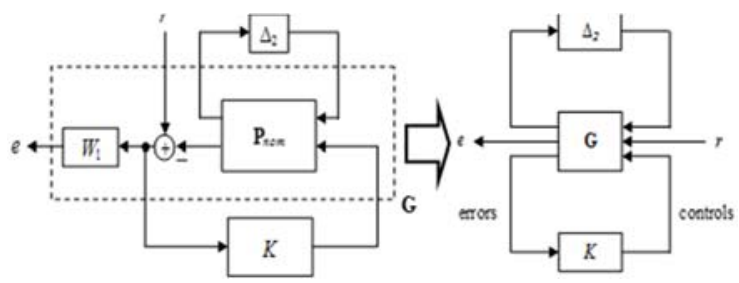

Figure 2: Closed-loop interconnection.

Plant (4) and Figure 1 can be transformed to the interconnections in Figure 2 with the sensitivity function as a performance indicator and $\mathbf{P}_{\text {nom }}$ being open-loop interconnection from Figure 1.

Perturbation matrix has the form:

$$
\begin{gathered}
\Delta_{2} \equiv\left[\begin{array}{ccc}
\Delta_{a} & 0 & 0 \\
0 & \Delta_{b} & 0 \\
0 & 0 & \delta_{d e l}
\end{array}\right] \\
\Delta_{a} \equiv\left[\begin{array}{cccc}
\delta_{a 0} & 0 & \ldots & 0 \\
0 & \delta_{a 1} & \cdots & 0 \\
\vdots & \vdots & \ddots & \vdots \\
0 & 0 & \ldots & \delta_{a n-1}
\end{array}\right] \\
\Delta_{b} \equiv\left[\begin{array}{cccc}
\delta_{b 0} & 0 & \ldots & 0 \\
0 & \delta_{b 1} & \ldots & 0 \\
\vdots & \vdots & \ddots & \vdots \\
0 & 0 & \ldots & \delta_{b n}
\end{array}\right]
\end{gathered}
$$

For stability and performance Theorem 1 and the following Corollary 1 hold:

Theorem 1: For $\Delta$ defined by (9) the loop in Figure 2 is wellposed, internally stable and $\left\|\mathbf{F}_{L}\left[\mathbf{F}_{U}\left(\mathbf{G}, \Delta_{2}\right), K\right]\right\|_{\infty} \leq 1$ if and only if

$$
\sup _{\substack{\omega \in R \\ K \text { stabilizing } \mathbf{G}}} \mu_{\Delta}\left[\mathbf{F}_{L}(\mathbf{G}, K)(j \omega)\right] \leq 1
$$

with $\boldsymbol{\Delta} \equiv\left\{\left[\begin{array}{cc}\delta_{1} & 0 \\ 0 & \Delta_{2}\end{array}\right], \delta_{P} \in \mathbf{C}\right\}$.

Proof: The proof is the same as in Doyle et al., 1982 and Packard and Doyle, 1993 except for the fact that perturbations are complex matrices which simplifies the proof and complies with the definition of $\mu$ (Definition 1).

Define sensitivity function as transfer function from reference $r$ to error $e$ in Figure 3:

$$
S \equiv \frac{1}{1+P K}
$$

Now, as a consequence of Theorem 1, a suffi-cient condition for the robust stability and perfor-mance of the feedback loop in Figure 3 can be formed for sensitivity function $S$ and family of plants (4).

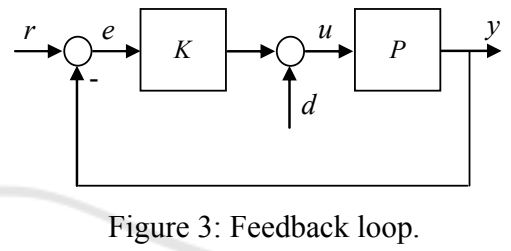

Corollary 1: For the set of plants (4), the feedback loop in Figure 3 is internally stable and $\left\|S W_{1}\right\|_{\infty} \leq 1$ if and only if (12), (5), (6), (7) and (8) hold.

Proof: The proof follows from Figure 1, inequalities (12), (5), definitions (6), (7) and (8) and Theorem 1.

\section{ALGEBRAIC $\mu$-SYNTHESIS}

The algebraic $\mu$-synthesis can be applied to any control problem that can be transformed to the loop in Figure 2 where $\mathbf{G}$ denotes the generalized plant, $\mathbf{K}$ is the controller, $\Delta_{d e l}$ is the perturbation matrix, $r$ is the reference and $e$ is the output.

For the purposes of the algebraic $\mu$-synthesis, the MIMO system with $l$ inputs and $l$ outputs has to be decoupled into $l$ identical SISO plants. The nominal model is defined in terms of transfer functions:

$$
\mathbf{P}_{n o m}(s) \equiv\left[\begin{array}{ccc}
P_{11}(s) & \cdots & P_{1 l}(s) \\
\vdots & \ddots & \vdots \\
P_{l 1}(s) & \cdots & P_{l l}(s)
\end{array}\right]
$$

For decoupling the nominal plant $\mathbf{P}_{\text {nom }}\left(\mathbf{P}_{\text {nom }}\right.$ invertible) it is satisfactory to have the controller in the form 


$$
\mathbf{K}(s)=K(s) \mathbf{I}_{l} \operatorname{det}\left[\mathbf{P}_{n o m}(s)\right] \frac{1}{P_{x y}(s)}\left[\mathbf{P}_{n o m}(s)\right]^{-1}
$$

where $P_{x y}$ is an element of $\operatorname{adj}\left[\mathbf{P}_{\text {nom }}(s)\right]=\operatorname{det}\left[\mathbf{P}_{\text {nom }}(s)\right]$. $\cdot\left[\mathbf{P}_{\text {nom }}(s)\right]^{-1}$ with the highest degree of numerator $\left\{\operatorname{adj}\left[\mathbf{P}_{\text {nom }}(s)\right]\right.$ denotes adjugate matrix of $\left.\mathbf{P}_{\text {nom }}\right\}$. The choice of the decoupling matrix prevents the controller from cancelling any poles or zeros from the right half-plane so that internal stability of the nominal feedback loop is held. The MIMO problem is reduced to finding a controller $K(s)$ which is tuned via setting the poles of the nominal feedback loop with the plant

$$
\begin{aligned}
\mathbf{P}_{\text {dec }}(s) & =\frac{1}{P_{x y}(s)} \operatorname{det}\left[\mathbf{P}_{n o m}(s)\right]\left[\mathbf{P}_{n o m}(s)\right]^{-1} \mathbf{P}_{n o m}(s) \\
& =\frac{1}{P_{x y}(s)} \operatorname{det}\left[\mathbf{P}_{n o m}(s)\right] \mathbf{I}_{l}
\end{aligned}
$$

Define

$$
P_{d e c} \equiv \frac{1}{P_{x y}(s)} \operatorname{det}\left[\mathbf{P}_{n o m}(s)\right]
$$

Transfer function $P_{d e c}$ can be approximated by a system $P_{d e c}^{*}$ with lower order than $P_{d e c}$

$$
P_{d e c}^{*}(s)=\frac{b(s)}{a(s)}
$$

which can be rewritten in terms of its coefficients and transformed to the elements of $\mathbf{R}_{\mathrm{PS}}$

$$
P_{\text {dec }}^{*}(s)=\frac{\frac{b_{0}+b_{1} s+\ldots+b_{n} s^{n}}{\frac{\left(\alpha_{1}+s\right)\left(\alpha_{2}+s\right) \cdot \ldots \cdot\left(\alpha_{n}+s\right)}{s^{n}+a_{0}+a_{1} s+\ldots+a_{n-1} s^{n-1}}}}{\left(\alpha_{1}+s\right)\left(\alpha_{2}+s\right) \cdot \ldots \cdot\left(\alpha_{n}+s\right)}=\frac{B}{A}, A,
$$

The controller $K=N_{K} / D_{K}$ is derived as solution of the Diophantine equation

$$
A D_{K}+B N_{K}=1
$$

with $A, B, D_{K}, N_{K} \in \mathbf{R}_{\mathrm{PS}}$. Equation (20) is the Bezout identity. All feedback controllers $N_{K} / D_{K}$ are given by

$$
K=\frac{N_{K}}{D_{K}}=\frac{N_{K_{0}}-A T}{D_{K_{0}}+B T}, N_{K_{0}}, D_{K_{0}} \in \mathbf{R}_{\mathrm{PS}}
$$

where $N_{K_{0}}, D_{K_{0}} \in \mathbf{R}_{\mathrm{PS}}$ are particular solutions of (20) and $T$ is an arbitrary element of $\mathbf{R}_{\mathrm{PS}}$.

The controller $K$ satisfying equation (20) guarantees the BIBO (bounded-input bounded-output) stability of the feedback loop in Figure 4. This is a crucial point for the theorems regarding the structured singular value. If the BIBO stability is held, then the nominal model is internally stable and theorems related to robust stability and performance can be used. The BIBO stability also guarantees stability of $\mathbf{F}_{L}(\mathbf{G}, \mathbf{K})$ making possible usage of performance weights with integration property implying non-existence of state space solutions using DGKF formulae (see Doyle et al., 1989) due to zero eigenvalues of appropriate Hamiltonian matrices. This procedure results in zero steady-state error in the feedback loop with the controller obtained as a solution to equation (20) being neither possible in the scope of the standard $\mu$-synthesis using DGKF formulae, nor using LMI approach (see Gahinet and Apkarian, 1994) leading to numerical problems in most of realworld applications.

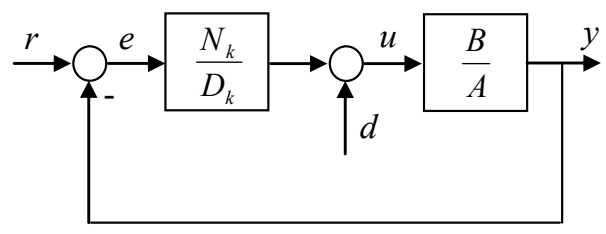

Figure 4: Nominal feedback loop.

The aim of global optimization in the algebraic approach is to design a controller satisfying the condition:

$$
\begin{aligned}
& \sup \mu_{\Delta}\left[\mathbf{F}_{L}(\mathbf{G}, \mathbf{K})\left(\omega, \alpha_{1}, \ldots, \alpha_{n+n_{1}+n_{2}}, t_{1}, \ldots, t_{n_{2}}\right)\right] \leq 1, \omega \in( \\
& \infty,+\infty)
\end{aligned}
$$

where $n+n_{1}+n_{2}$ is the order of the nominal feedback system, $n_{1}$ is the order of particular solution, $K_{0}, t_{i}$ are arbitrary parameters

$T=\frac{t_{0}+t_{1} s+\ldots+t_{n_{2}} s^{n_{2}}}{\left(\alpha_{n_{1}+1}+s\right) \cdot \ldots \cdot\left(\alpha_{n_{1}+n_{2}}+s\right)}$ and $\mu_{\Delta}$ denotes the structured singular value of LFT on generalized plant $\mathbf{G}$ and controller $\mathbf{K}$ with $\boldsymbol{\Delta}$ defined in (12).

Tuning parameters are positive and constrained to the real axis since parameters of the transfer function have to be real and due to the fact that com-plex poles cause oscillations of the nominal feed-back loop.

A crucial problem of the cost function in (22) is the fact that many local extremes are present. Hence, local optimization does not yield a suitable or even stabilizing solution. This can be overcome via evolutionary computation solving the task very efficiently.

\section{EXAMPLE - PROBLEM FORMULATION \& SOLUTION}

The problem to solve is $2^{\text {nd }}$ order system with $1^{\text {st }}$ order 
astatism and uncertain time delay:

$$
\begin{gathered}
\mathbf{P} \equiv\left\{\frac{b_{0}}{a_{2} s^{2}+a_{1} s} e^{-\tau s}\right\} \\
a_{1} \in[1.8,2.2], a_{2}=1, \\
b_{0} \in[1.8,2.2], \tau \in[0,4]
\end{gathered}
$$

The control objective is to find a controller for which the robust stability and performance is held for every plant from the set $\mathbf{P}$. The weights follow from (6) and (7):

$$
W_{a 1}=0.2, W_{b 0}=0.2
$$

The time delay is treated by multiplicative uncertainty (see Figure 1)

$$
\left\{P\left(1+W_{d e l} \delta_{d e l}\right):\left|\delta_{d e l}\right| \leq 1, \delta_{d e l} \in \mathbf{C}\right\}
$$

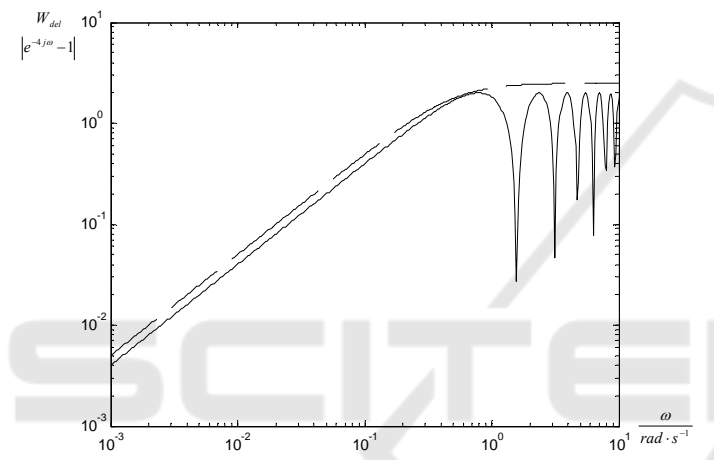

Figure 5: Bode plot of $W_{d e l}$ and $\left|e^{-4 j \omega}-1\right|$.

Let the nominal plant be

$$
P(s)=\frac{b}{a}=\frac{2}{s^{2}+2 s}
$$

then for the weighting function $W_{d e l}$ the following inequality must be held $\mathbf{P}^{\prime}$ being the set $\mathbf{P}$ omitting the parametric uncertainties

$$
\left|\frac{P^{\prime}(j \omega)}{P(j \omega)}-1\right|<\left|W_{d e l}(j \omega)\right|, \forall \omega \in \overline{\mathbf{R}}_{+}, \forall P^{\prime} \in \mathbf{P}
$$

which is equivalent with

$$
\left|e^{-\tau j \omega}-1\right|<\left|W_{d e l}(j \omega)\right|, \forall \omega \in \overline{\mathbf{R}}_{+}, \tau \in\left[0 ; T_{d}\right]
$$

The weight $W_{d e l}$ is defined as an envelope curve of $\left|e^{-\tau j \omega}-1\right|$. For $\tau=4, W_{d e l}$ can have the Bode plot depicted in Figure 5:

$$
W_{d e l}(s)=2.5 \frac{2 s}{2 s+1}
$$

The performance condition is of the form:

$$
\left\|W_{1} S\right\|_{\infty}<1
$$

where $S$ is the sensitivity function and weight $W_{1}$ (see Figure 2) is defined for the algebraic approach and $D$ $K$ iteration as follows:

$$
\begin{gathered}
W_{1}^{A}(s)=\frac{0.004}{10 s^{3}+100 s^{2}+s+10^{-5}} \cdot 50 \\
W_{1}^{D-K}(s)=\frac{0.004}{10 s^{3}+100 s^{2}+s+10^{-5}} \cdot 50
\end{gathered}
$$

By the optimization of the poles $\alpha_{i}$ via the Differential Migration and subsequent tuning by the NelderMead simplex method, resulting poles were obtained:

$\alpha_{1}=0.065, \alpha_{2}=0.063, \alpha_{3}=2.021, \alpha_{4}=62.338$

yielding the controller

$$
K_{A}(s)=\frac{4.618 s^{2}+8.182 s+0.2571}{s^{2}+62.49 s}
$$

The controller obtained from the $D-K$ iteration was approximated by the $3^{\text {rd }}$ order transfer function:

$$
K_{D-K}(s)=\frac{0.332 s^{3}+0.968 s^{2}+0.0442 s+0.0003}{s^{3}+8.926 s^{2}+0.0692 s+6.998 \cdot 10^{-7}}
$$

The $\mu$-plot in Figure 8 shows that both control-lers have the supremum of $\mu$ below one and the ro-bust stability and performance condition is satisfied.

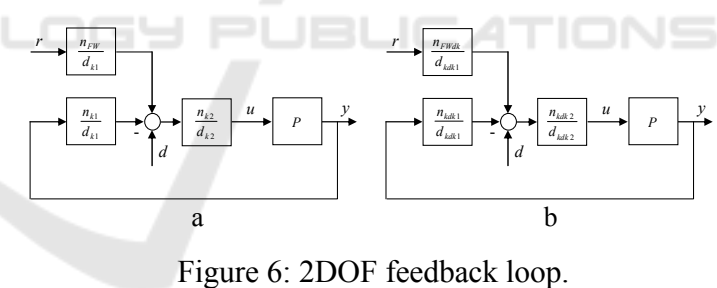

The controllers for 2DOF feedback loop (Figure 6a, $6 \mathrm{~b}$ - algebraic approach and $D-K$ iteration, respectively) have the compensator $\left(n_{k 2}, d_{k 2}, n_{k d k 2}, d_{k d k 2}\right)$ defined as fraction of the factors corresponding with most stable zero and least stable pole of $K_{A}$ and $K_{D-K}$ and feedback $\left(n_{k 1}, d_{k 1}, n_{k d k 1}, d_{k d k 1}\right)$ and feed-forward $\operatorname{part}\left(n_{F W}, d_{k l}, n_{F W}, d_{k d k 1}\right)$ defined by the fraction of the factors corresponding with remaining zeros and poles of $K_{A}$ and $K_{D-K}$ with $n_{F W}=n_{k 1}^{0}$ and $n_{F W d k}==n_{k d k 1}^{0}$ $n_{k 1}^{0}, n_{k d k 1}^{0}$ being the coefficients of $n_{k l}$ and $n_{k d k l}$ of zero exponent of $s$ ):

$$
\begin{aligned}
& \frac{n_{k 1}}{d_{k 1}}=\frac{4.618 s+0.1478}{s^{2}+62.49 s}, \frac{n_{F W}}{d_{k 1}}=\frac{0.1478}{s^{2}+62.49 s}, \frac{n_{k 2}}{d_{k 2}}=\frac{s+1.74}{s} \\
& \frac{n_{k k k 1}}{d_{k \alpha k 1}}=\frac{0.3316 s^{2}+0.0154 s+9.13 \cdot 10^{-5}}{s^{2}+8.926 s+0.0691} \quad, \quad \frac{n_{F W d k}}{d_{k k k 1}}=\frac{9.13 \cdot 10^{-5}}{s^{2}+8.926 s+0.0691}, \\
& \frac{n_{\text {hak } 2}}{d_{\text {kdk2 } 2}}=\frac{s+2.87}{s+1 \cdot 10^{-5}}
\end{aligned}
$$


The periodicity is defined via sinusoids changing the uncertain parameters in the intervals defined by (23):

$$
b_{0}=\bar{b}_{0}\left[1+\lambda_{b_{0}} \sin \left(\omega_{b_{0}} t\right)\right]
$$

where $\bar{b}_{0}=2 \quad \lambda_{b_{0}}=0.2$ and $\omega_{b_{0}}=0.1$. The step response for the periodic change (38) is in Figure 7.

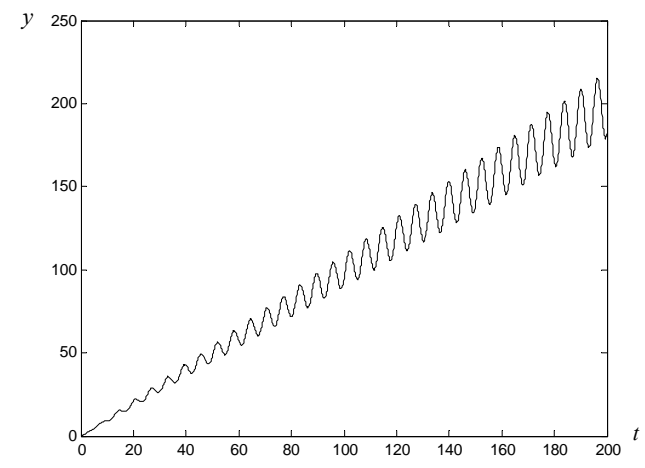

Figure 7: Step response for periodic changes of parameters.

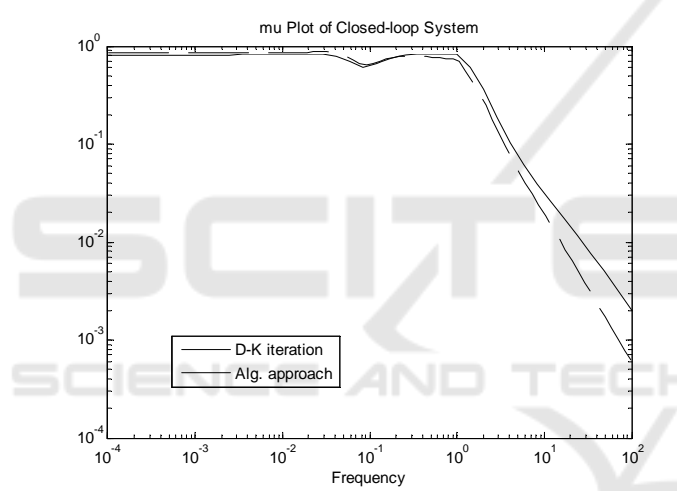

Figure $8: \mu$-plots for the controllers obtained by the $D-K$ iteration and algebraic approach

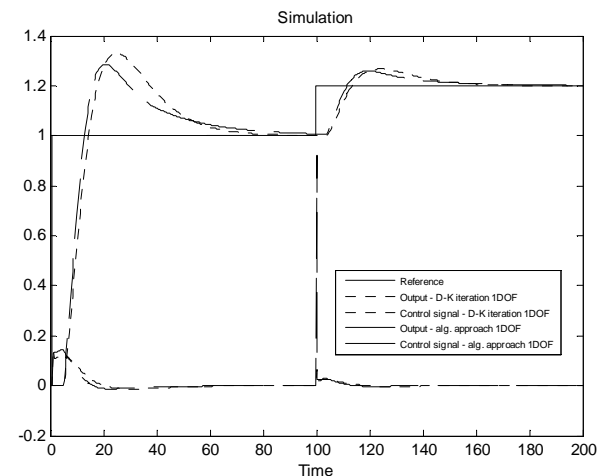

Figure 9: Simulations for simple feedback loop without periodic changes - algebraic approach and $D-K$ iteration.

Simulations in Figure 9 and 10 show that $D-K$ iteration yields non-zero steady-state error in contrast to the algebraic approach having no steady state error and faster set point tracking than the $D-K$ iteration controller. Simulations for periodic changes (38) in Figure 11 and 12 prove that the $1 \mathrm{DOF}$ and $2 \mathrm{DOF}$ feedback loops are stable for both the algebraic approach and $D-K$ iteration. In all simu-lations full time delay is connected, i.e. $\tau=4 \mathrm{~s}$.

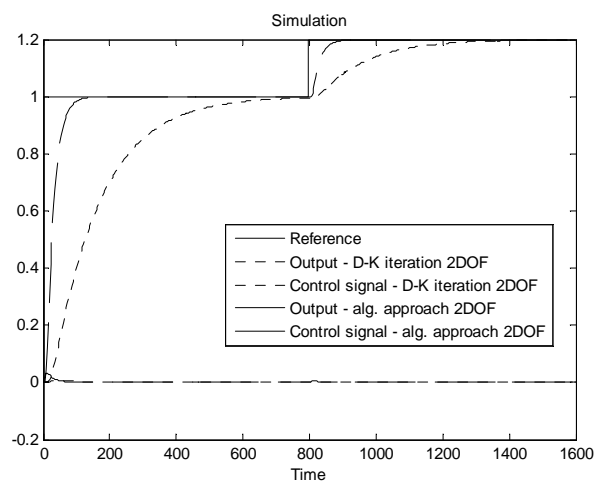

Figure 10: Simulations for 2DOF feedback loop without periodic changes - algebraic approach and $D-K$ iteration.

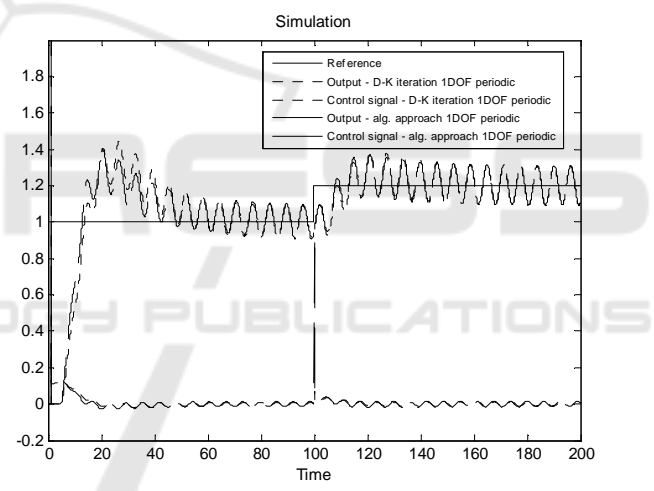

Figure 11: Simulations for simple feedback loop with periodic changes - algebraic approach and $D-K$ iteration.

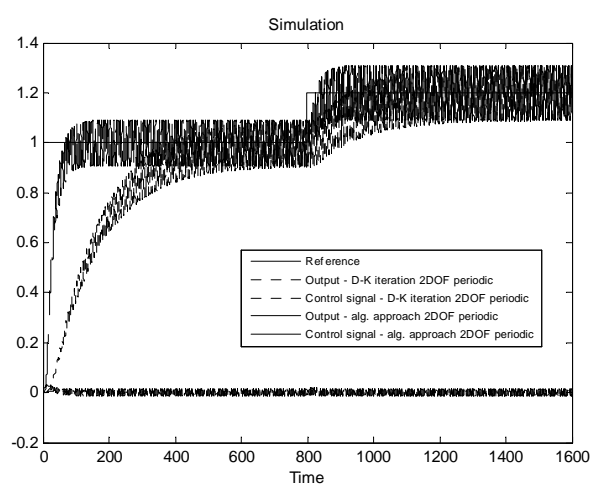

Figure 12: Simulations for 2DOF feedback loop with periodic changes - algebraic approach and $D-K$ iteration. 


\section{USER INTERFACE}

The main window of the Matlab toolbox consists of three sections (see Figure 13):

- System Definition

- Controller Design

- Simulation and Verification

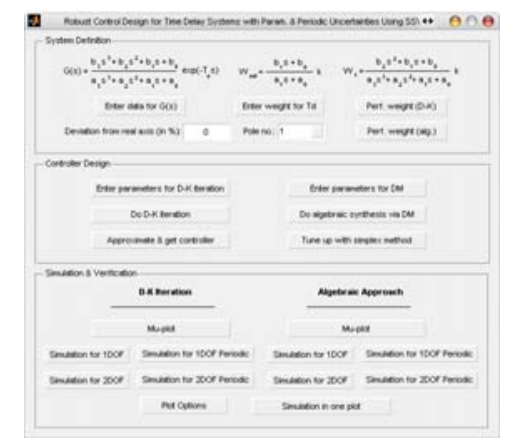

Figure 13: The main window.

\subsection{System Definition}

System definition has the button for displaying the dialog for entering parameters of the control plant where the parameters of transfer function, the maximum value of time delay and parameters for periodic changes can be entered (Figure 14).

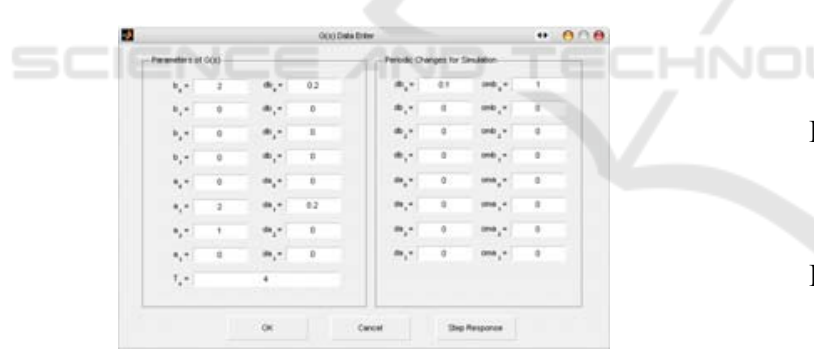

Figure 14: Dialog for entering parameters of the controlled plant.

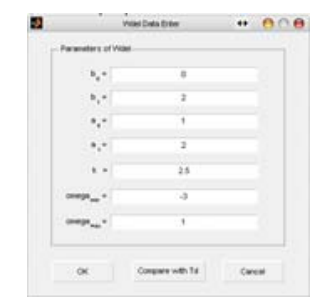

Figure 15: Dialog for entering the parameters of the weight $W_{\text {del. }}$.

Another button displays the dialog for entering the parameters of the weight $W_{d e l}$ treating uncertain time delay (Figure 15) with button showing the Bode plot of the weight $W_{d e l}$ compared to the left side of (28).

In the last part of system definition, buttons showing dialogs for entering parameters of the performance weight $W_{1}$ are placed. There are separate weights for the $D-K$ iteration and algebraic approach. Each dialog has a button for showing the Bode plot of the particular weight.

\section{ACKNOWLEDGEMENTS}

This work was supported by the Ministry of Education, Youth and Sports of the Czech Republic within the National Sustainability Programme project No. LO1303 (MSM - 7778/2014).

\section{REFERENCES}

Barmish, B. R., 1984. Invariance of strict Hurwitz property for polynomials with perturbed coefficients, IEEE Transactions on Automatic Control, Vol. 29.

Barmish, B. R., 1989. A generalization of Kharitinov's four polynomial concept for robust stability with linearly depen-dent coefficient perturbations, IEEE Transactions on Automatic Control, Vol. 34, No. 2.

Barmish, B., Ackermann, J.E., Hu, H.Z., 1989. The tree struc-tured decomposition: a new approach to robust stability analysis, Proceedings Conference on Information Sciences and Systems, John Hopkins University, Baltimore.

Barmish, B., Shi, Z., 1990. Robust stability of class of polyno-mials with coefficient depending multilinearly on perturba-tions, IEEE Transactions on Automatic Control, Vol. 35, No. 9.

Bartlett, A. C., Hollot, C., Lin, H., 1988. Root Locations of an entire polytope of polynomials: it suffices to check the edges, Mathematics of Control, Signals and Systems, Vol. 1, No. 1, pp. 61-71.

Bialas, S., 1983. A necessary and sufficient conditions for stability of interval matrices, International Journal of Control, Vol. 37, pp. 717-722.

Chapellat, H., Bhattacharyya, S.P., 1989. An alternative proof of Kharitonov's theorem: robust stability of interval plants, IEEE Transactions on Automatic Control, Vol. 34, No. 3.

Chapellat, H., Dahleh, M., Bhattacharyya, S.P., 1993. Robust stability manifolds for multilinear interval systems, IEEE Transactions on Automatic Control, Vol. 38, No. 2.

Dlapa, M., 2011. "Controller Design for a Two Tank System Using Structured Singular Value and Direct Search Methods," 18th World Congress of the International Federation of Automatic Control (IFAC), August 28 - September 2, Milan, Italy, pp. 7511-7516, ISSN: $1474-6670$ 
Dlapa, M., 2014. "Air-Heating Set Control via Direct Search Method and Structured Singular Value," 13th European Control Conference (ECC 2014), June 2427, Strasbourg, France, pp. 600-605, ISBN 978-39524269-2-0.

Dlapa, M., 2017. „Cluster Restarted DM: New Algorithm for Global Optimisation“. Intelligent Systems Conference 2017 (IntelliSys 2017), September 7-8, London, UK, pp. 1130-1135, ISBN 978-1-5090-64359.

Dlapa, M., 2018. „General Parametric and Periodic Uncertainties and Time Delay Robust Control Design Toolbox, "IEEE The 19th International Conference on Industrial Technology (IEEE ICIT 2018), February 2022, Lyon, France, pp. 181-186, ISBN 978-1-50905948-5.

Doyle, J. C., Wall, J., Stein, G., 1982. "Performance and robust-ness analysis for structured uncertainty," in Proceedings of the 21st IEEE Conference on Decision and Control, pp. 629-636.

Doyle, J. C., 1985. "Structure uncertainty in control system design," in Proceedings of 24th IEEE Conference on decision and control, pp. 260-265.

Doyle, J. C., Khargonekar, P. P., Francis, B.A., 1989. "State-space solutions to standard $\mathrm{H} 2$ and $\mathrm{H} \infty$ control problems," IEEE Transactions on Automatic Control, vol. 34 , no. 8, pp. 831-847.

Packard, A., Doyle, J. C., 1993. "The complex structured singular value," Automatica, vol. 29(1), pp. 71-109.

Fu, M., Dasgupta, S., Blondel, V., 1995. "Robust stability under a class of nonlinear parametric perturbations," IEEE Transactions on Automation Control, Vol. 40, No. 2.

Gahinet, P., Apkarian, P., 1994. "A linear matrix inequality app-roach to $\mathrm{H} \infty$ control," International Journal of Robust and Nonlinear Control, 4, 421-449.

Kharitonov, V., 1978. Asymptotic stability of an equilibrium position of a family of linear differential equations, Differencialnye Uravneniya, Vol. 14.

Packard, A., Doyle, J. C., 1993. "The complex structured singular value," Automatica, vol. 29(1), pp. 71-109.

Sideris, A., de Gaston, R., 1986. Multivariable stability margin calculation with uncertainty correlated parameters, Proceedings Conference on Decision and Control, Athens.

Stein, G., Doyle, J., 1991. "Beyond Singular Values and Loop-shapes," AIAA Journal of Guidance and Control, Vol. 14, No. 1, pp. 5-16.

Zadeh, L., Desoer, C., 1963. Linear System Theory: The State Space Approach, McGraw-Hill, New York.

Internet: http://dlapa.cz/homeeng.htm 